\title{
PROBLEMES POSES PAR L'ELABORATION D'UN DICTIONNAIRE JURIDIQUE AU CANADA
}

\author{
E. Groffler et D. ReEd
}

Tout rédacteur utilise des outils de travail qui comprennent, bien sar, des dictionnaires de langue. Quand il lui faut rédiger dans un domaine spécialisé, s'ajoutent a ses outils des dictionnaires, des glossaires, des vocabulaires couvrant le vocabulaire appartenant aux domaines des connaissances dont il est question dans le texte à rédiger. En droit, et surtout en common law, l'usage des dictionnaires et des vocabulaires bilingues ou unilingues anglais est général. C'est pourquoi il nous a paru essentiel de nous pencher sur la question des qualites que l'usager serait a même d'exiger d'un ouvrage de ce genre.

En octobre 1986, nous avions propose au Conseil de recherches en sciences humaines du Canada de subventionner une recherche intitulee $A$ la rechenche d'un dictionnaire de droit dans un systeme juridique bilingue. Cette demande agréée, nous avons décidé d'examiner, dans une première partie, la clientele, le corpus et le dépouillement, le choix des entrés et les définitions.

La deuxième partie sera consacrée aux problèmes particuliers posés par le dictionnaire bilingue. Dans les deux cas, nous nous proposons d'examiner le roble que peut, et pourquoi pas que doit, jouer l'informatique. Une troisieme et dernière partie résumera les éléments des deux premières pour en tirer des conclusions applicables plus particulierement au contexte canadien. Nous ne couvrirons que la première partic ici.

Si les dictionnaires de langue ont fait l'objet d'études lexicographiques sérieuses et diverses, ${ }^{1}$ les dictionnaires de specialité sont les parents pauvres du domaine. Seul ressort le Vocabulaire de l'astronautique de Louis Gilbert. ${ }^{2}$ Ce dernier ouvrage n'est d'ailleurs pas a proprement parler une étude de lexicographie. Il constitue plutôt une enquête sur le vocabulaire de l'astronautique dans la presse écrite entre le 12 avril 1961 (vol de Gagarine) et le 14 juin 1963 (vol conjoint de Valérie Bikovski et de Valentina Terchkova). L'objet de l'ouvrage n'était pas d'analyser le vocabulaire de l'astronautique ni de faire un dictionnaire, mais de dresser l'état de ce vocabulaire a un moment donne, sous l'angle particulier de la diffusion de masse. 
Il nous a semblé opportun de remédier a cette carence et ce, pour diverses raisons. Tout d'abord, les ouvrages en cours d'élaboration au Canada, auxquels les auteurs participent ou ont participe a un titre ou a un autre, peuvent éclairer nos recherches et également en bénéficier. Ensuite, le caractère bijuridique et bilingue du droit du Canada en fait le laboratoire rêvé pour l'étude des problèmes tant d'un dictionnaire définitoire que d'un dictionnaire bilingue.

Nous en sommes toujours à l'étape d'élaboration de notre recherche. Notre intention, ici, est de vous donner un aperçu de notre premier chapitre consacré a la notion de dictionnaire. Les deuxième et troisième chapitres traiteront respectivement du contenu des entrées (la définition, la forme que doit prendre l'entrée de dictionnaire, la nature, le caractère normatif éventuel, les références et les renvois) et des moyens utilisés pour rédiger un dictionnaire (l'équipe des rédacteurs et des chercheurs ainsi que l'usage de l'informatique).

Ainsi, le premier chapitre de notre monographie sera consacré a la notion même de dictionnaire, c'est-à-dire à ses destinataires (le public-cible), au corpus a partir duquel il est établi, a la manière d'effectuer le depouillement et au choix des entrees. Un de nos premiers soucis a été de définir ce que nous entendions par "dictionnaire" et de tâcher de le différencier des glossaires, lexiques et autres vocabulaires. Nous utilisons le terme "dictionnaire" dans son sens usuel, et la definition du Grand Larousse de la langue française ${ }^{3}$ nous convient parfaitement: "Recueil des mots et des expressions d'une langue ou d'une catégorie d'entre eux, généralement rangés par ordre alphabétique (selon un ordre différent, mais permettant de les retrouver aisément) et suivis de leur définition dans cette langue ou de leur traduction dans une langue étrangère." Quant au dictionnaire de spécialité, il y est plus particulièrement défini comme un "ouvrage relatif à une matière, une science, un art, et dont les articles sont disposés par ordre alphabétique." L'expression "dictionnaire de droit" ou "law dictionary" nous semble ainsi appropriée. Cependant, il nous faut noter l'expression souvent employée de "vocabulaire juridique." Le doyen Cornu l'a choisie à la suite de Henri Capitant en grande partie, nous a-t-il dit, pour garder le même titre que son prédécesseur et aussi pour éviter les confusions avec bon nombre d'ouvrages qui n'ont de dictionnaire que le nom. Le terme "vocabulaire juridique" est d'ailleurs également approprié et le Grand Larousse de la langue française ${ }^{4}$ donne comme emploi actuel sous l'entré "vocabulaire": "Dictionnaire où ne sont relevés et définis que les mots appartenant a une langue spéciale, généralement une langue technique: vocabulaire de la musique, de la chimie et du cinéma." Nous pouvons également citer le titre de l'ouvrage d'André Lalande: Vocabulaire technique et critique de la philosophie.5 Cependant, 
Littre ${ }^{6}$ n'est pas tout a fait d'accord sur ce point quand il dit sous l'entrée "vocabulaire": "Vocabulaire et glossaire ne s'appliquent guere qu'a des purs dictionnaires de mots au lieu que "dictionnaire," en genéral, comprend non seulement les dictionnaires de langue mais encore les dictionnaires historiques et ceux des sciences et des arts." Le dictionnaire Littre nous a paru vieilli sur cette question. Le Robert, ${ }^{7}$ lui, semble abonder dans le sens du Larousse. Nous n'avons pas encore effectué de recherche analogue en anglais.

Le dictionnaire de droit revêt évidemment des caractères propres. Dans son article intitule "Lexicography and Interpretation of Law," Thomas Gizbert Studnicki-Krakow nous dit que le lexicographe qui rédige un dictionnaire de langue considere "the corpus of utterances as a sample of an unlimited set of potential utterances," tandis que "the corpus of utterances analyzed by the jurist is a finite set of valid legal regulations."

Enfin, il faut mentionner la distinction existant entre "dictionnaire" et "encyclopédie", c'est-à-dire entre le "dictionnaire des mots" et le "dictionnaire des choses." Un dictionnaire de droit, même s'il s'en defend, a toujours quelque peu un caractere encyclopedique. Il est parfois difficile de ne pas entrer dans le fonctionnement de l'institution a definir, et cela est tout simplement impossible quand le dictionnaire doit, par exemple, expliquer aux civilistes une institution de common law et aux juristes de droit anglais une institution de droit civil. A ce sujet, nous vous renvoyons à l'excellente introduction du Dirionario Giuridico--Inglese-Italiano/Law Dictionary--EnglishItalian' et nous vous invitons a penser aux explications que devrait contenir un dictionnaire de traduction comme celui de Doucet, ${ }^{10}$ par exemple.

Nous en sommes arrivés la conclusion que le dictionnaire de droit, tel que nous l'envisageons, devrait être présenté selon l'ordre alphabétique des entrées. Les dictionnaires thématiques sont intéressants, certes, mais a condition qu'existent deja des dictionnaires alphabétiques. En effet, le classement par themes exige une profonde connaissance de ceux-ci pour retrouver les notions voulues. En outre, dans un dictionnaire qui serait totalement bilingue, un tel classement serait susceptible de devenir un véritable casse-tête.

Dans sons avis "aux lecteurs" du Tresor de la langue française,"1 Paul Imbs dit que "la premiere tâche d'un lexicographe est d'esquisser le profil de son public." Il faut donc déterminer les categories de lecteurs auxquels s'adresse un dictionnaire de droit. Si l'on pense au dictionnaire idéal-étant bien entendu qu'il n'existe pas-il devrait satisfaire les avocats, les juges, les professeurs de droit, les étudiants, les traducteurs et le public en genéral. 
La difficulté évidente d'un dictionnaire de spécialité est qu'il s'adresse à plusieurs catégories trés différentes d'usagers. Il s'agit de déterminer les besoins spécifiques de ces différentes catégories et de se demander si les membres d'une de ces catégories n'excluent pas nécessairement ceux des autres. Nous pouvons utiliser ici l'exemple d'un étudiant qui cherche dans un dictionnaire de droit des réponses à de multiples questions qui peuvent aller de la définition d'un mot n'appartenant pas au vocabulaire usuel jusqu'au résumé d'un cas célèbre en passant par l'explication d'une institution ou la biographie d'un juriste d'autrefois. The Oxford Companion to Law ${ }^{12}$ constitue sans contredit le meilleur exemple d'un tel ouvrage et l'une des plus précieuses mines d'information juridique qui soient. Le traducteur ou le rédacteur "parallèle" (c'est-à-dire celui qui élabore son texte dans une langue en même temps que cela est fait dans l'autre langue par un autre rédacteur), au contraire, ira plus volontiers vers un ouvrage lui donnant la définition succincte et la traduction d'un mot; le Vocabulaire de la common law $w^{13}$ répond assez bien à leurs besoins. Ainsi, ils y apprendront rapidement, dans le tome I, que "equitable conversion" se dit "conversion en Equité" en français et signifie la "transformation par laquelle, en Equité, un bien réel est considéré comme personnel ou un bien personnel comme réel." On peut se demander également si l'étymologie ou les expressions latines qui semblent indispensables a un professeur de droit présentent un intérêt quelconque pour les traducteurs, pour les rédacteurs ou pour le grand public. Des traducteurs nous ont dit que la première qualité qu'ils attendaient l'un dictionnaire était la possibilité de le consulter rapidement; ils n'aiment pas y voir des détails inutiles comme l'étymologie des mots ou des références. La pression a laquelle ils sont si souvent soumis explique cette réaction qui est néanmoins regrettable.

Il est évident que la clientèle-cible d'un dictionnaire influence sa nature. Le dictionnaire définitoire unilingue est plus utile aux étudiants et au grand pubic, le lexique bilingue au traducteur. Cependant, ainsi que nous l'avons dit plus haut, la nature bi-systémique du droit canadien rend inévitable dans un dictionnaire juridique général (par opposition à un dictionnaire strictement limité à une discipline du droit: droit aérien, droit criminel...) l'explication de certaines institutions connues dans un des systèmes et inconnues dans l'autre, d'où un certain caractère encyclopédique du dictionnaire.

On peut également s'interroger sur l'intérêt que présente un dictionnaire de droit pour le grand public, du moins un dictionnaire de droit complet. Si certains lexiques contenant des définitions se veulent simples et facilement utilisables pour "éviter cette rupture que provoque parfois dans la lecture d'un passage juridique, d'un article de journal ou de revue, la 
parution d'un mot, d'une formule dont le sens est plus connu ou totalement ignoré du lecteur,"14 un ouvrage vraiment technique fait appel à des notions complexes et sa lecture devient plus ardue pour le profane. Si le Vocabulaire juridique de Henri Capitant ${ }^{15}$ s'adressait aussi au "public composé de non-juristes" en 1936, le nouveau Vocabulaire juridique de Gérard Cornu ${ }^{16}$ se borne a être destiné à "tout juriste, étudiant, chercheur, praticien," sans pour cela mentionner le grand public. Le fait que des lecteurs non ou moins spécialisés ont recours de temps a autre au dictionnaire tend a gonfler le nombre des entrées sans égard a la fréquence d'usage. II nous semble en tous cas que leurs besoins devraient être satisfaits premièrement par les dictionnaires de langue généraux et ne devrajent pas influencer les choix à faire dans la rédaction d'un ouvrage destiné aux spécialistes (rétention du latin, par exemple).

Passons à présent à l'étude du corpus. Il est évident que nous appelons ainsi le recueil d'énoncès trouvés lors du dépouillement. $\mathrm{Si}$, dans le Vocabulaire juridique de Henri Capitant, on avait procédé au "dépouillement méthodique des tables des grands ouvrages, des répertoires, des dictionnaires," l'introduction du Vocabulaire juridique de Gérard Cornu se borne a mentionner la reprise d'un certain nombre de termes qui figuraient dans le premier vocabulaire. En fait, selon le doyen Cornu, la même méthode a été utilisée et les spécialistes chargés des différentes matières ont contribué a déterminer la liste des termes, liste qui a constamment ét enrichie au fur et à mesure des travaux. Au Canada, la préface du Lexique anglais/français du droit en Ontario ${ }^{17}$ explique que la terminologie est celle qui figure dans la loi de l'Ontario, dans certaines lois fédérales comme le Code criminel et que les auteurs ont pu puiser dans les vocabulaires normalisés du Programme de l'administration de la justice dans les deux langues officielles. Le corpus du Vocabulaire de la common law, ${ }^{18}$ publié par Moncton, varie évidemment d'après le thème de chaque volume et il n'a pas toujours été exactement défini dans l'introduction. En règle générale, il s'agit des lois du Nouveau-Brunswick, des jugements traduits du Nouveau-Brunswick, de lois fédérales et de lois du Manitoba. En outre, il y a eu dépouillement de certains textes de doctrine comme, par exemple, The Law of Torts de Fleming pour le tome IV (sur les delits civils). Cette brève énumération montre au moins que la composition du corpus varie d'un ouvrage à l'autre et que le souci de le déterminer d'une façon vraiment scientifique a peu préoccupé les lexicographes. Il nous semble qu'idéalement il faudrait un corpus pleinement représentatif des disciplines couvertes, déterminé dans le temps et facile a tenir a jour.

La première question a se poser est de savoir si les dictionnaires font partie du corpus. Il est certain que c'est le cas lorsqu'il est question d'un 
dictionnaire de langue, puisqu'il s'agit alors, d'apres le Trésor de la langue française, ${ }^{19}$ d'ouvrages-témoins de l'usage et que du seul fait d'avoir été "lus ou consultés, ils ont agi sur la langue, soit en la stabilisant ou en en freinant l'évolution, soit en incitant a l'utilisation de matériaux qui acquéraient une autorité du seul fait qu'ils étaient accueillis par les lexicographes." Quand il s'agit d'un dictionnaire specialisé, par contre, on se doit de poser cette question sérieusement. Le caractère incomplet des dictionnaires existants au Canada et celui peu adéquat des dictionnaires publiés a l'étranger, du point de vue du droit canadien, pourraient constituer des motifs qui justifieraient leur exclusion. En revanche, toute utilisation d'index que l'auteur d'un dictionnaire n'a pas établi lui-même présente inévitablement des lacunes par rapport a un dépouillement systématique de textes. Il nous parait vain, cependant, d'avoir toujours a tout refaire.

Un dictionnaire juridique doit être fondé sur les termes utilisés dans la législation et il est pratique de faire usage des index de cette législation. Etant donné qu'un dictionnaire canadien complet devrait représenter tant le droit civil que la common law, il faudrait naturellement se servir du Code civil et du Code de procédure civile du Québec ainsi que de toutes les lois fédérales. Cela ne suffirait d'ailleurs pas car, au Québec par exemple, des matières importantes sont laissées à des lois particulières comme celle sur la protection du consommateur ou celle sur la protection de la jeunesse, et de larges portions du droit privé de common law sont laissées a la législation provinciale.

Etant donné que, pour être vraiment utile, un dictionnaire de droit canadien devrait être bilingue, on pourrait se servir de toute la législation bilingue existante, c'est-à-dire non seulement des lois fédérales mais encore des lois traduites du Nouveau-Brunswick, de l'Ontario, du Manitoba et bientôt des territoires du Nord-Ouest. Quant a la jurisprudence, pour limiter l'étendue du corpus et avoir une jurisprudence représentative de tout le Canada, il semblerait qu'il faille considérer, outre la jurisprudence de la Cour suprême, dont les arrêts sont traduits et équipés d'un index depuis 1970, celle de la Cour fédérale du Canada, ainsi que les jugements bilingues du Nouveau-Brunswick et des autres provinces où l'on traduit parfois des jugements considérés comme importants. Les sources que nous venons de décrire constituent une première "couche" du corpus, qui présente l'avantage d'être bien délimitée, de pouvoir être complétée et d'être objective. Elle ne contient cependant pas certains termes historiquement valables, comme par exemple, "concubin." II faudra donc permettre a l'équipe des définisseurs de prendre l'initiative de proposer des ajouts au corpus. 
Une fois le corpus établi, il doit être examiné par une équipe de jurilinguistes (c'est-at-dire des juristes avec d'excellentes connaissances linguistiques et des linguistes avec d'excellentes connaissances juridiques) qui se trouveront en fait devant deux operations a accomplir: tout d'abord, il leur faudra examiner les index déja établis et, dans certains cas, remettre les mots dans leur contexte pour en tirer les sens différents pour ensuite dépouiller les textes pour lesquels n'existe aucun index. Ces deux tâches ne peuvent être accomplies que par des personnes extrémement qualifiees. Les lexicographes interrogés s'accordent pour dire que la tache du dépouillement ne peut être confiée qu'à des personnes tout à fait expérimentés.

Pour ce qui est d'un dictionnaire de droit, il faut qu'il porte sur les termes juridiques de la langue de base. Cette affirmation parast une lapalissade, mais nous verrons, a l'analyse, que le sens du mot "terme" comme celui du mot "juridique" gagne a être précisé. Premièrement, les définitions portent sur des mots. Dans l'introduction de son Vocabulaire juridique, Gárard Cornu déclare que "l'átude englobe toutes les combinaisons de termes qui, se situant au même niveau que le mot comme elément linguistique indivisible, ont la meme fonction d'unite significative élémentaire." 20 Il s'agit aussi des mots composés et des syntagmes consactés. Les auteurs du Vocabulaire ont retenu comme entrées principales les syntagmes qui présentaient une "individualité linguistique suffisante par rapport a leurs composantes." Les syntagmes, d'ailleurs, quoique moins stables que les mots composes, sont indispensables dans un dictionnaire de spécialité. Dans ce méme ordre d'idees, Thomas Gizbert Studnicki-Krakow fait remarquer: "A structure containing a pause is considered to be one word if its meaning had become lexicalized, that is if it does not depend entirely on the meanings of its components and on the way of their combination. For instance, the structure 'legal person' is considered as one word, because its meaning is not determined by the meanings of the word 'legal' and 'person' and by the relevant syntactic rule. The structure as a whole can be ascribed a lexical meaning." ${ }^{21}$ Il semble meme qu'il faille aller un peu plus loin et donner les syntagmes essentiels a l'utilisation, en droit, d'un grand nombre de termes juridiques. La majorite des dictionnaires ignore trop souvent cet aspect particulier de l'utilisation d'une langue specialisée. Il n'est pas suffisant d'avoir trouve le mot action (dans son sens d'action en justice), dans un texte juridique, il est tout aussi nécessaire de savoir que l'on dit, en droit, intenter une action et non pas **faire une action, **tengager une action, ou encore ***prendre une action, mais que l'on peut dire engager une instance et introduire une instance. Les locutions verbales, tout en ne constituant pas la partie essentielle du lexique, doivent egalement y trouver leur place. Elles ne s'arretent pas non plus au verbe lui-méme; l'objet est également important et le juriste doit savoir que l'on dit poursuivre 
quelqu'un, mais intenter une action contre quelqu'un, pour ne citer que deux exemples tirés du vocabulaire de la procédure civile. Une difficulté fondamentale dans le choix de termes juridiques durant le dépouillement est qu'un mot de la langue générale placé dans un environnement spécifique contient parfois une charge sémantique juridique. Cest le cas pour les adjectifs gratui, immédiat, immémorial grave, caché, par exemple, dans les expressions acte à titre gratuit cause inmétiate, usage inménorial, faute grave, vice cache. La place de la locution dans l'ordre alphabétique dépend, comme l'affirme Gérard Cornu de "la plus forte attraction sémantique."2 A la difference de ce qui se fait dans certains dictionnaires scientifiques, il est difficile dans des disciplines comme le droit de se limiter aux noms ou aux locutions nominales. Tant le Dictionnaire de droit prive ${ }^{23}$ de McGill que le Vocabulaire juridique de Gérard Cornu ${ }^{24}$ déclarent innover en introduisant des verbes et des adjectifs dans le dictionnaire. En fait, déjà dans le vocabulaire de Henri Capitant, ${ }^{25}$ on trouve "germain" et "utérin" qu'il eut été tout de même difficile de rattacher à un substantif. Il est sage de ne pas s'en tenir uniquement aux entrées nominales, d'autant plus que les adjectifs, les adjectifs polysémiques en particulier, revêtent en droit une grande importance. Gerard Cornu nous dit d'ailleurs dans son introduction que 'l'éminence de leur fonction, dans le vocabulaire juridique, vient sans doute en compensation du nombre limité des termes de celui-ci." ${ }^{126}$

Contrairement a de nombreux jurilinguistes, Georges Mounin pense qu"'au sens propre, il n'existe pas de langue du droit en soi mais seulement, à l'intérieur de la langue française, un vocabulaire du droit, et sans doute quelques tours syntaxiques spécifiques."27 Le professeur Mounin nous semble simplifier grandement le probleme. Le langage du droit, nous l'avons dit ailleurs, ${ }^{23}$ se rapproche davantage des langues de spécialité qui tendent à purifier leur lexique, leur syntaxe et leur sémantique de manière à réaliser des énoncés dénués d'équivoque. Elles essaient d'y arriver en remplaçant les mots intuitifs et imagés de la langue courante par des termes abstraits et rigoureusement définis, qui excluent toute ambiguité. Studnicki-Krakow souligne très justement la difference qui existe entre le lexicographe et le juriste. D'apres lui, l'un et l'autre expliquent la signification des mots en les definissant, mais le lexicographe qui ródige un dictionnaire de langue ne tient pas compte de tous les mots et de toutes les expressions, étant donné que certains de ces mots, que certaines de ces expressions ne sont tout simplement pas acceptables. Le juriste, lui, n'a pas le choix; il ne peut se permettre d'ecarter certains mots ou certaines expressions qui sont contenues dans la loi ou dans la jurisprudence. Toujours d'apress Studnicki-Krakow, il doit tenir compte de la personne qui a formulé tel mot ou telle expression et il lui faut répondre à une question inconnue du lexicographe ordinaire: "What is the relation of the language used by the legislator to the natural language used in a community submitted to a given system of law?'zo 
De nombreux mots du langage naturel peuvent bien sûr se charger d'un sens juridique. Si l'on écarte les simples faits, méme quand ils ont des conséquences juridiques--comme c'est le cas pour la plupart des événements -la juridicité leur vient, selon Gerard Cornu, si "les traits de leur nature auxquels le droit attache des effets répondent a des conditions que pose le droit et donc à une notion juridique qui leur confère un sens au regard du droit." ${ }^{30}$ Ainsi, l'erreur est instituée par la loi comme vice du consentement, a condition qu'elle présente certaines caractéristiques. ${ }^{31}$ Cette méthode ne doit pas être absolument rigoureuse et il faut, comme le dit Henri Capitant, "corriger l'esprit de géométrie par l'esprit de finesse." ${ }^{132}$

Alain Rey, quant a lui, fait remarquer que, dans les vocabulaires de spécialité, la sélection sémantique et pragmatique ne saurait être rigoureusement distinguée et que, par exemple, un dictionnaire de marine décrit les unités lexicales utilisées par les gens de mer tout en éliminant les éléments qui sont communs a ceux-ci et a d'autres locuteurs comme, notamment, mer, nuage, vent. Cependant, il ajoute que le choix depend des options précédentes du modele, de la constitution du corpus et des criteres sélectifs des auteurs. ${ }^{33}$ En outre, la notion même de "vocabulaire" d'une spécialité est assez relative car, comme l'a dit R. L. Wagner, "part faite aux nomenclatures et aux traits/tiques qui colorent les phraséologies, des expressions telles que vocabulaire politique, vocabulaire mathématique, vocabulaire religieux sont des lieux communs de valeur tres faible, sinon nulle. Les mathématiciens, les théologiens utilisent quand il faut des termes hautement techniques, univoques (autant que se peut), mais ces mots et ces lexiques ne constituent pas le tout d'une discussion sur l'efficacite du khi ou sur la grâce suffisante."134

Les critères de choix sont évidemment tres subtils et difficiles a manier. David Mellinkoff reproche aux dictionnaires de droit de contenir de nombreux mots qui n'ont aucune raison d'y être, c'est-à-dire des mots de la langue ordinarie qui ne possèdent pas de sens différent en droit que dans la langue générale. Il cite, par exemple, proper, improper, garden, horse power, sex et il ajoute: "casual involvement with the law is a poor criterion of selection for law dictionaries." 35

Enfin, certains termes sont en voie de disparition. Que l'on pense a "concubin" remplacé par "conjoint de fait" ou a "droits de l'homme" qui fait place a "droits de la personne." II est important cependant que ces termes "historiques" figurent dans le dictionnaire car ils existent encore en doctrine et servent a la compréhension de l'évolution du droit. 
Une fois le choix des entrées établi, il s'agit de procéder à leur définition. Les problèmes que soulève la définition ont été évoqués, entre autres, lors d'un colloque qui s'est tenu à Québec en $1982^{36}$ ainsi que par Paul Imbs: ${ }^{37}$ "... si la définition doit informer sur la substance sémique du mot de manière à permettre à celui-ci de désigner déictement le référé translinguistique qui est l'objet final de tout discours humain, elle doit aussi et surtout viser à ce que cette désignation renvoie au référé distinctement, c'est-a-dire sans confusion avec un autre référé et donc sans confusion avec un autre mot: la spécificité, c'est-à-dire l'indication du ou des traits distinctifs est sa règle principale." L'étude de l'élaboration de la définition fait l'objet du chapitre suivant de notre recherche et constitue une étape encore plus ardue que la première.

\section{NOTES}

${ }^{1}$ Voir, par exemple pour la France, Lucien Collignon et Michel Glatigny, Les dictionnaires, initiation à la lexicographie, Paris, CEDIC, 1978; J. Dubois et C.L. Dubois, Introduction a la lexicographie, Paris, Larousse, 1971; Josette Rey-Debove, Lexique sémiotique, Paris, Presses universitaires de France, 1979.

${ }^{2}$ Louis Guilbert, Vocabulaire de l'astronautique, 1967.

${ }^{3}$ Grand Larousse de la langue française, Paris, Larousse, 1978.

${ }^{4}$ Op. cit.

${ }^{5}$ André Lalande, Vocabulaire technique et critique de la philosophie, Paris, Presses universitaires de France, 1976.

${ }^{6}$ Emile Littré, Dictionnaire de la langue française, Paris Gallimard/Hachette, 1964-1966.

${ }^{7}$ Paul Robert, Le Grand Robert de la langue française. Dictionnaire alphabétique et analogique de la langue française, Paris, Le Robert, 1985.

${ }^{8}$ Thomas Gizbert Studnicki-Krakow, "Lexicography and Interpretation of Law", 1977, XIIII/L, Archives de philosophie du droit et de la philosophie sociale 161, p. 169.

${ }^{9}$ Francesco D. Franchis, Dizionario Giuridico-Inglese-Italiano/Law Dictionany--English-Italian, Milan, Giuffre, 1984, surtout a la page 29. 
${ }^{10}$ Michel Doucet, Dictionnaire juridique et économique. Français-anglais et anglais-français, Paris, La Maison du Dictionnaire, 1979.

${ }^{11}$ Trésor de la langue française. Dictionnaire de la langue du 19 et du 20e siecle, Paris, C.N.R.S., 1971-.

12D.M. Walker, The Oxford Companion to Law, Oxford, Clarendon Press, 1980.

${ }^{13}$ Centre de traduction et de terminologie juridique, Vocabulaire de la "common law", Les Editions du Centre universitaire de Moncton:

Tome I, Droit des biens. Procédure civile, 1980.

Tome II, Droit des fiducies, 1982.

Tome III, Procédure civile. Preuve, 1983.

Tome IV, Délits civils, 1986.

${ }^{14}$ Raymond Guillen et Jean Vincent, Lexique de termes juridiques, Paris, Dalloz, 1978, p. III.

${ }^{15}$ Henri Capitant, Vocabulaire juridique, Paris, Presses universitaires de France, 1936.

${ }^{16}$ Gérard Cornu, Vocabulaire juridique, Paris, Association Henri Capitant, Presses universitaires de France, 1987.

${ }^{17}$ Lexique anglais/français du droit en Ontario, Toronto, Ministère du Procureur général, 1987.

${ }^{18}$ Op. cit.

${ }^{19}$ Op. cit., p. 37.

${ }^{20}$ Op. cit. p. VIII.

${ }^{21}$ Op. cit., p. 165 .

220 p. cit., p. 6.

${ }^{23}$ Dictionnaire de droü prive, Centre de recherche en droit privé et comparé du Québec, 1985, p. V. 
${ }^{24}$ Op. cit.

25Op. cit.

${ }^{26}$ Op. cit.

${ }^{27}$ Georges Mounin, "La linguistique comme science auxiliaire dans les disciplines juridiques", 1974, 19 Archives de philosophie du droit, p. 11 et 12.

${ }^{28}$ David G. Reed, "Une fiche terminologique juridique", 1983, Multilingua 101, p. 170.

${ }^{29}$ Op. cit.

${ }^{30}$ Op. cit., p. IX.

${ }^{31}$ Gérard Cornu, op. cit., sous erreur.

32Op. cit., p. 6.

${ }^{33}$ Alain Rey, "Typologie des dictionnaires", 1970, Langages, $\mathrm{n}^{0} 19,48$, p. 55.

${ }^{34}$ R.-L. Wagner, "Réflexions nafves à propos des dictionnaires", 1975, 11 Cahier de Lexicologie, p. 96.

${ }^{35}$ David Mellinkoff, "The Myth of Precision and the Law Dictionary", 1983, 31 U.C.L.A. Law Review 423, p. 436.

${ }^{36}$ TERMIA, Problèmes de la définition et de la synonymie en terminologie, Actes du Colloque international de terminologie, Université Laval, Quebec, 23-27 mai 1982, Québec, Gisterm, OLF, DGTD, 1983.

${ }^{37}$ Paul Imbs, op. cit.

E. Groffier est Professeur, Faculté de droit, McGill Universiny.

D. Reed est Professeur, Ecole de traduction, College universitaire de SaintBoniface, University of Manitoba. 